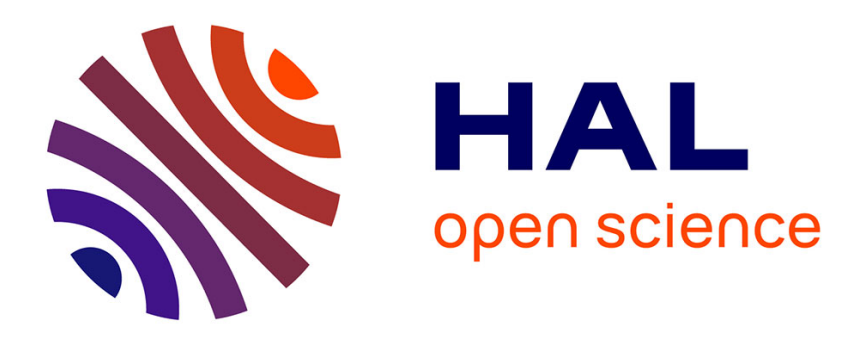

\title{
Morse-Sard type results in sub-Riemannian geometry
}

Ludovic Rifford, Emmanuel Trélat

\section{To cite this version:}

Ludovic Rifford, Emmanuel Trélat. Morse-Sard type results in sub-Riemannian geometry. Mathematische Annalen, 2005, 332 (1), pp.145-159. hal-00086340

\section{HAL Id: hal-00086340 \\ https://hal.science/hal-00086340}

Submitted on 18 Jul 2006

HAL is a multi-disciplinary open access archive for the deposit and dissemination of scientific research documents, whether they are published or not. The documents may come from teaching and research institutions in France or abroad, or from public or private research centers.
L'archive ouverte pluridisciplinaire HAL, est destinée au dépôt et à la diffusion de documents scientifiques de niveau recherche, publiés ou non, émanant des établissements d'enseignement et de recherche français ou étrangers, des laboratoires publics ou privés. 


\title{
Morse-Sard type results in sub-Riemannian geometry
}

\author{
L. Rifford, E. Trélat ${ }^{\dagger}$
}

\begin{abstract}
Let $(M, \Delta, g)$ be a sub-Riemannian manifold and $x_{0} \in M$. Assuming that Chow's condition holds and that $M$ endowed with the subRiemannian distance is complete, we prove that there exists a dense subset $N_{1}$ of $M$ such that for every point $x$ of $N_{1}$, there is a unique minimizing path steering $x_{0}$ to $x$, this trajectory admitting a normal extremal lift. If the distribution $\Delta$ is everywhere of corank one, we prove the existence of a subset $N_{2}$ of $M$ of full Lebesgue measure such that for every point $x$ of $N_{2}$, there exists a minimizing path steering $x_{0}$ to $x$ which admits a normal extremal lift, is nonsingular, and the point $x$ is not conjugate to $x_{0}$. In particular, the image of the sub-Riemannian exponential mapping is dense in $M$, and in the case of corank one is of full Lebesgue measure in $M$.
\end{abstract}

\section{Introduction and main results}

The following general definition of a sub-Riemannian distance (also called CarnotCarathéodory distance) is due to [3]. Let $M$ be a connected smooth $n$-dimensional manifold, $m$ an integer such that $1 \leqslant m \leqslant n$, and $f_{1}, \ldots, f_{m}$ be smooth vector fields on the manifold $M$. For all $x \in M$ and $v \in T_{x} M$, set

$$
g(x, v):=\inf \left\{\sum_{i=1}^{m} u_{i}^{2} \mid u_{1}, \ldots, u_{m} \in \mathbb{R}, \sum_{i=1}^{m} u_{i} f_{i}(x)=v\right\} .
$$

Then $g(x, \cdot)$ is a positive definite quadratic form on the subspace of $T_{x} M$ spanned by $f_{1}(x), \ldots, f_{m}(x)$. Outside this subspace we set $g(x, v)=+\infty$. The form $g$ is called sub-Riemannian metric associated to the $m$-tuple of vector fields $\left(f_{1}, \ldots, f_{m}\right)$. Let $\mathcal{A} C([0,1], M)$ denote the set of absolutely continuous paths in $M$ defined on $[0,1]$, we define the length of $\gamma \in \mathcal{A} C([0,1], M)$ as

$$
l(\gamma):=\int_{0}^{1} \sqrt{g(\gamma(t), \dot{\gamma}(t))} d t .
$$

\footnotetext{
*Institut Girard Desargues, Université Lyon I, 43 Bd du 11 Novembre 1918, 69622 Villeurbanne cedex, France. E-mail: rifford@igd.univ-lyon1.fr.

$\dagger$ Univ. Paris-Sud, Lab. AN-EDP, Math., UMR 8628, Bat. 425, 91405 Orsay Cedex, France. E-mail: emmanuel.trelat@math.u-psud.fr.
} 
We say that Chow's condition holds if the Lie algebra spanned by the vector fields $f_{1}, \ldots, f_{m}$, is equal to the tangent space $T_{x} M$ at every point $x$ of $M$. It is well-known that under this condition any two points of $M$ can be joined by an absolutely continuous path with finite length.

The sub-Riemannian distance associated to the $m$-tuple of vector fields $\left(f_{1}, \ldots, f_{m}\right)$, between two points $x_{0}, x_{1}$ in $M$, is defined as

$$
d_{S R}\left(x_{0}, x_{1}\right):=\inf \left\{l(\gamma) \mid \gamma \in \mathcal{A} C([0,1], M), \gamma(0)=x_{0}, \gamma(1)=x_{1}\right\} .
$$

The sub-Riemannian sphere $S_{S R}\left(x_{0}, r\right)$ (resp. the sub-Riemannian ball $B_{S R}\left(x_{0}, r\right)$ ) centered at $x_{0}$ with radius $r$ as the set of points $x \in M$ such that $d_{S R}\left(x_{0}, x\right)=r$ (resp. $\left.d_{S R}\left(x_{0}, x\right)<r\right)$. A path $\gamma \in \mathcal{A C}([0,1], M)$ is said to be minimizing if it realizes the sub-Riemannian distance between its extremities.

Remark 1.1. If Chow's condition holds, then:

- the topology defined by the sub-Riemannian distance $d_{S R}$ coincides with the original topology of $M$,

- sufficiently near points can be joined by a minimizing path,

- if the manifold $M$ is moreover a complete metric space for the sub-Riemannian distance $d_{S R}$, then any two points can be joined by a minimizing path.

Consider on the other part the differential system on the tangent bundle $T M$ of $M$

$$
\dot{x}(t)=\sum_{i=1}^{m} u_{i}(t) f_{i}(x(t)) \quad \text { a.e. on }[0,1]
$$

where the function $u(\cdot)=\left(u_{1}(\cdot), \ldots, u_{m}(\cdot)\right)$, called control function, belongs to $L^{2}\left([0,1], \mathbb{R}^{m}\right)$. Let $x_{0} \in \mathbb{R}^{n}$, and let $\mathcal{U}$ denote the (open) subset of $L^{2}\left([0,1], \mathbb{R}^{m}\right)$ such that the solution of (1) starting at $x_{0}$ and associated to a control $u(\cdot) \in \mathcal{U}$ is well-defined on $[0,1]$. The mapping

$$
\begin{aligned}
E_{x_{0}}: \mathcal{U} & \longrightarrow \mathbb{R}^{n} \\
u(\cdot) & \longmapsto x(1),
\end{aligned}
$$

which to a control $u(\cdot)$ associates the extremity $x(1)$ of the corresponding solution $x(\cdot)$ of (1) starting at $x_{0}$, is called end-point mapping at the point $x_{0}$; it is a smooth mapping. The trajectory $x(\cdot)$ is said to be singular if the associated control $u(\cdot)$ is a singular point of the end-point mapping (i.e. if the Fréchet derivative of $E_{x_{0}}$ at $u(\cdot)$ is not onto); it is minimizing if it realizes the sub-Riemannian distance between its extremities.

Remark 1.2. A sub-Riemannian manifold is often defined as a triple $(M, \Delta, g)$, where $M$ is a $n$-dimensional manifold, $\Delta$ is a distribution of rank $m \leqslant n$, and $g$ is a Riemannian metric on $\Delta$. If the vector fields $\left(f_{1}, \ldots, f_{m}\right)$ are everywhere linearly independent, then controlled paths solutions of (1) coincide with absolutely continuous paths tangent to the distribution $\Delta$, where

$$
\Delta(x)=\operatorname{Span}\left\{f_{1}(x), \ldots, f_{m}(x)\right\}
$$


for all $x \in M$. These paths are said $\Delta$-horizontal.

On the other part, for $x_{0} \in M$, let $\Omega\left(x_{0}, \Delta\right)$ be the set of $\Delta$-horizontal paths starting from $x_{0}$ whose derivative is square integrable for the metric $g$ (and hence for any Riemannian metric on $\Delta$ ). Endowed with the $H^{1}$-topology, $\Omega\left(x_{0}, \Delta\right)$ inherits of a Hilbert manifold structure, see [4]. For $\left(x_{0}, x_{1}\right) \in M \times M$, let $\Omega\left(x_{0}, x_{1}, \Delta\right)$ be the subset of paths $x(\cdot) \in \Omega\left(x_{0}, \Delta\right)$ such that $x(1)=x_{1}$. The set $\Omega\left(x_{0}, x_{1}, \Delta\right)$ is a submanifold of $\Omega\left(x_{0}, \Delta\right)$ in a neighborhood of any nonsingular path, but might fail to be a (global) manifold due to the possible existence of singular paths.

Let $x_{0}$ and $x_{1}$ in $M$. The sub-Riemannian problem of determining a minimizing trajectory steering $x_{0}$ to $x_{1}$ can be easily seen (up to reparametrization, and using the Cauchy-Schwarz inequality) to be equivalent to the optimal control problem of finding a control $u(\cdot) \in \mathcal{U}$ such that the solution of the control system (1) steers $x_{0}$ to $x_{1}$ in time 1 , and minimizes the cost function

$$
C(u(\cdot)):=\int_{0}^{1} \sum_{i=1}^{m} u_{i}(t)^{2} d t
$$

If a control $u(\cdot)$ associated to a trajectory $x(\cdot)$ such that $x(0)=x_{0}$ is optimal, then there exists a nontrivial Lagrange multiplier $\left(\psi, \psi^{0}\right) \in T_{x(1)}^{*} M \times \mathbb{R}$ such that

$$
\psi \cdot d E_{x_{0}}(u(\cdot))=-\psi^{0} d C(u(\cdot)),
$$

where $d E_{x_{0}}(u(\cdot))$ (resp. $\left.d C(u(\cdot))\right)$ denotes the Fréchet derivative of $E_{x_{0}}$ (resp. $C$ ) at the point $u(\cdot)$. The well-known Pontryagin maximum principle (see [8]) parametrizes this condition and asserts that the trajectory $x(\cdot)$ is the projection of an extremal, that is a quadruple $\left(x(\cdot), p(\cdot), p^{0}, u(\cdot)\right)$, solution of the constrained Hamiltonian system

$$
\begin{gathered}
\dot{x}(t)=\frac{\partial H}{\partial p}\left(x(t), p(t), p^{0}, u(t)\right), \dot{p}(t)=-\frac{\partial H}{\partial x}\left(x(t), p(t), p^{0}, u(t)\right), \\
\frac{\partial H}{\partial u}\left(x(t), p(t), p^{0}, u(t)\right)=0,
\end{gathered}
$$

almost everywhere on $[0,1]$, where

$$
H\left(x, p, p^{0}, u\right):=\left\langle p, \sum_{i=1}^{m} u_{i} f_{i}(x)\right\rangle+p^{0} \sum_{i=1}^{m} u_{i}^{2}
$$

is the Hamiltonian of the optimal control problem, $p(\cdot)$ (called adjoint vector) is an absolutely continuous mapping on $[0,1]$ such that $p(t) \in T_{x(t)}^{*} M$, and $p^{0}$ is a real nonpositive constant. Moreover there holds

$$
\left(p(1), p^{0}\right)=\left(\psi, \psi^{0}\right),
$$

up to a multiplying scalar. If $p^{0}<0$ then the extremal is said to be normal, and in this case it is normalized to $p^{0}=-1 / 2$. If $p^{0}=0$ then the extremal is said to be abnormal. 
Remark 1.3. Any singular trajectory is the projection of an abnormal extremal, and conversely.

Furthermore, a singular trajectory is said to be strict (or strictly singular) if it does not admit a normal extremal lift; equivalently in that case we say that its abnormal extremal lift is strictly abnormal.

The sub-Riemannian wave-front $W_{S R}\left(x_{0}, r\right)$ centered at $x_{0}$ and with radius $r$ is defined as the set of end-points $x(1)$, where $\left(x(\cdot), p(\cdot), p^{0}, u(\cdot)\right)$ is an extremal such that $x(0)=x_{0}$ and $C(u(\cdot))=r^{2}$. Under Chow's condition, it is clear from Remark 1.1 that $S_{S R}\left(x_{0}, r\right)$ is a subset of $W_{S R}\left(x_{0}, r\right)$.

Using the previous normalization, controls associated to normal extremals can be computed as

$$
u_{i}(t)=\left\langle p(t), f_{i}(x(t)\rangle, i=1, \ldots, m .\right.
$$

Hence normal extremals are solutions of the Hamiltonian system

$$
\dot{x}(t)=\frac{\partial H_{1}}{\partial p}(x(t), p(t)), \dot{p}(t)=-\frac{\partial H_{1}}{\partial x}(x(t), p(t)),
$$

where

$$
H_{1}(x, p)=\frac{1}{2} \sum_{i=1}^{m}\left\langle p, f_{i}(x)\right\rangle^{2} .
$$

Notice that $H_{1}(x(t), p(t))$ is constant along each normal extremal and that the length of the path $x(\cdot)$ equals $\left(2 H_{1}(x(0), p(0))\right)^{1 / 2}$. Actually, given a point $x_{0}$ of $M$, the differential system (5) has a well-defined smooth solution on $[0,1]$ such that $x(0)=x_{0}$ and $p(0)=p_{0}$, for $p_{0} \in U$, where $U$ is a connected open subset of $T_{x_{0}}^{*} M$. In what follows, the point $x_{0}$ is fixed.

Definition 1.1. The smooth mapping

$$
\begin{aligned}
\exp _{x_{0}}: U & \longrightarrow M \\
p_{0} & \longmapsto x(1)
\end{aligned}
$$

where $(x(\cdot), p(\cdot))$ is the solution of the system (5) such that $x(0)=x_{0}$ and $p(0)=p_{0}$, is called exponential mapping at the point $x_{0}$.

The exponential mapping parametrizes normal extremals. Notice that every minimizing trajectory steering $x_{0}$ to a point of $M \backslash \exp _{x_{0}}(U)$ is necessarily strictly singular.

Remark 1.4. Using notations of Definition 1.1, it is easy to see by reparametrization that $x(t)=\exp _{x_{0}}\left(t p_{0}\right)$, for all $t \in[0,1]$.

Remark 1.5. For all $p_{0} \in U$ such that $H_{1}\left(x_{0}, p_{0}\right)=\frac{r^{2}}{2}$, one has $\exp _{x_{0}}\left(p_{0}\right) \in$ $W_{S R}\left(x_{0}, r\right)$. The space of normal extremals with length $r$ is parametrized by the manifold $U_{r}=U \cap H_{1}^{-1}\left(\frac{r^{2}}{2}\right)$, which is diffeomorphic to $\mathcal{S}^{m-1} \times \mathbb{R}^{n-m}$ if the distribution $\Delta$ has rank $m$ at $x_{0}$. 
A point $x \in \exp _{x_{0}}(U)$ is said conjugate to $x_{0}$ if it is a critical value of the mapping $\exp _{x_{0}}$, i.e. if there exists $p_{0} \in U$ such that $x=\exp _{x_{0}}\left(p_{0}\right)$ and the differential $d \exp _{x_{0}}\left(p_{0}\right)$ is not onto. The conjugate locus, denoted by $\mathcal{C}\left(x_{0}\right)$, is defined as the set of all points conjugate to $x_{0}$.

Remark 1.6. By Sard Theorem applied to the mapping $\exp _{x_{0}}$, it is clear that the conjugate locus $\mathcal{C}\left(x_{0}\right)$ has Lebesgue measure zero in $M$.

Remark 1.7. Let $x \in \exp _{x_{0}}(U)$, and $p_{0} \in U$ such that $x=\exp _{x_{0}}\left(p_{0}\right)$. We denote by $\left(x\left(\cdot, p_{0}\right), p\left(\cdot, p_{0}\right),-\frac{1}{2}, u\left(\cdot, p_{0}\right)\right)$ the associated normal extremal. Then we have

$$
\exp _{x_{0}}\left(p_{0}\right)=E_{x_{0}}\left(u\left(\cdot, p_{0}\right)\right) .
$$

Therefore if $x$ is not conjugate to $x_{0}$ then the control $u\left(\cdot, p_{0}\right)$ is nonsingular. In particular, the set of endpoints of nonstrictly singular trajectories starting from $x_{0}$ has Lebesgue measure zero in $M$.

Remark 1.8. With notations of the previous remark, if $x$ is not conjugate to $x_{0}$ then the path $x(\cdot):=x\left(\cdot, p_{0}\right)$ associated to the control $u(\cdot):=u\left(\cdot, p_{0}\right)$ admits a unique normal extremal lift. Indeed if it had two distinct normal extremals lifts $\left(x(\cdot), p_{1}(\cdot),-\frac{1}{2}, u(\cdot)\right)$ and $\left(x(\cdot), p_{2}(\cdot),-\frac{1}{2}, u(\cdot)\right)$, then the extremal $\left(x(\cdot), p_{1}(\cdot)-\right.$ $\left.p_{2}(\cdot), 0, u(\cdot)\right)$ would be an abnormal extremal lift of the path $x(\cdot)$, which is a contradiction since $u(\cdot)$ is nonsingular.

In the present paper we prove the two following theorems.

Theorem 1.1. Suppose Chow's condition holds, and that the manifold $M$ is complete for the sub-Riemannian distance $d_{S R}$. There exists a dense subset $N_{1}$ of $M$ such that, for every point $x \in N_{1}$, there is a unique minimizing path joining $x_{0}$ to $x$; moreover this trajectory admits a normal extremal lift. In particular the image $\exp _{x_{0}}(U)$ of the exponential mapping is dense in $M$.

For all $x \in M$, let $\Delta(x):=\operatorname{Span}\left\{f_{1}(x), \cdots, f_{m}(x)\right\}$, and let $\mu$ denote the Lebesgue measure on $M$. Regarding the previous result, one can wonder whether almost every point of $M$ belongs to $\exp _{x_{0}}(U)$. The following result gives a positive answer in the case of a corank-one distribution.

Theorem 1.2. Suppose Chow's condition holds, and that the manifold $M$ is complete for the sub-Riemannian distance $d_{S R}$. If the distribution $\Delta$ is everywhere of corank one, then there exists a subset $N_{2}$ of $M$ of full Lebesgue measure such that, for every point $x \in N_{2}$, there exists a minimizing path joining $x_{0}$ to $x$ and having a normal extremal lift. Moreover this trajectory is nonsingular, and $x$ is not conjugate to $x_{0}$. In particular, the set $\exp _{x_{0}}(U)$ is of full measure in $M$, i.e. $\mu\left(M \backslash \exp _{x_{0}}(U)\right)=0$.

The next two sections are devoted to the proof of the latter results. In a last section we discuss some consequences and open problems. 


\section{Proof of Theorem 1.1}

\subsection{The proximal sub-differential}

Let $M$ be a smooth manifold of dimension $n$ and $\Omega$ be an open subset of $M$. Let $f: \Omega \rightarrow \mathbb{R}$ be a continuous function on $\Omega$; we call proximal sub-differential of the function $f$ at the point $x \in \Omega$ the subset of $T_{x}^{*} M$ defined by

$$
\partial_{P} f(x):=\left\{d \phi(x) \mid \phi \in C^{\infty}(M) \text { and } f-\phi \text { attains a local minimum at } x\right\} .
$$

Note that since every local $C^{\infty}$ function can be extended to a $C^{\infty}$ function on $M$, the proximal sub-differential of $f$ at $x$ depends only on the local behavior of the function $f$ near $x$. In addition, remark that $\partial_{P} f(x)$ is a convex subset of $T_{x}^{*} M$ which may be empty; for instance the proximal sub-differential of the real function $t \mapsto-|t|$ at $t=0$ is empty.

Remark 2.1. Notice that when $M=\mathbb{R}^{n}$, a vector $\zeta$ belongs to the proximal sub-differential of $f$ at a point $x$ if and only if there exists $\sigma$ and $\delta>0$ such that

$$
f(y)-f(x)+\sigma\|y-x\|^{2} \geqslant\langle\zeta, y-x\rangle, \quad \forall y \in x+\delta B .
$$

This is the usual definition of proximal sub-differentials in Hilbert spaces; we refer the reader to $[6]$ for further details on that subject.

In fact, an immediate application of the smooth variational principle of Borwein-Preiss (see [5]) implies the following result.

Theorem 2.1. The proximal sub-differential of a continuous function $f: \Omega \rightarrow$ $\mathbb{R}$ is nonempty on a dense subset of $\Omega$.

The proximal sub-differential of $f$ defines a multivalued mapping from $\Omega$ into the cotangent bundle $T^{*} M$. It is said to be locally bounded on $\Omega$ if for each $x \in \Omega$ there exists a neighborhood $\mathcal{V}$ of $x$ such that $\partial_{P} f(\mathcal{V})$ is relatively compact in $T^{*} M$. The following result is standard.

Proposition 2.2. The function $f$ is Lipschitz continuous on $\Omega$ if and only if the proximal sub-differentials of $f$ are locally bounded on $\Omega$.

Remark 2.2. Notice that the Fréchet (or viscosity) sub-differential of $f$ at $x$, defined by

$$
D^{-} f(x):=\left\{d \phi(x) \mid \phi \in C^{1}(M) \text { and } f-\phi \text { attains a local minimum at } x\right\},
$$

is larger than the proximal sub-differential, but in fact both notions coincide locally; we refer the reader to [6, Prop. 4.5 p. 138 , Prop. 4.12 p. 142] for a precise statement.

To conclude this preliminary section, we remark that there exists a complete calculus of proximal sub-differentials, one that extends all the theorems of the usual smooth calculus, see [6]. 


\subsection{Application to the proof of Theorem 1.1}

In what follows we denote $e(\cdot):=d_{S R}\left(x_{0}, \cdot\right)^{2}$.

Proposition 2.3. Let $x \in M$ such that $\partial_{P} e(x) \neq \emptyset$. Then there exists a unique minimizing path $x(\cdot)$ joining $x_{0}$ to $x$. Moreover for every $\zeta \in \partial_{P} e(x)$, the path $x(\cdot)$ admits a normal extremal lift $\left(x(\cdot), p(\cdot),-\frac{1}{2}, u(\cdot)\right)$ such that $p(1)=\frac{1}{2} \zeta$.

Proof. We adopt the following notation: for every control $u(\cdot) \in \mathcal{U}$, we denote by $x_{u}(\cdot)$ the trajectory solution of (1) associated to the control $u(\cdot)$ and such that $x_{u}(0)=x_{0}$. Let $x \in M$ and $\zeta \in \partial_{P} e(x)$. We first prove that every minimizing path steering $x_{0}$ to $x$ admits a normal extremal lift such that $p(1)=\frac{1}{2} \zeta$. Let $u(\cdot) \in \mathcal{U}$ be an optimal control such that the associated trajectory $x_{u}(\cdot)$ joins $x_{0}$ to $x$; there holds

$$
e(x)=\int_{0}^{1} \sum_{i=1}^{m} u_{i}(t)^{2} d t .
$$

On the other hand, since $\zeta \in \partial_{P} e(x)$, there exists a function $\phi$ of class $C^{\infty}$ with $d \phi(x)=\zeta$ and such that $e-\phi$ attains a local minimum at $x$. Thus there exists a neighborhood $\mathcal{V}$ of $u(\cdot)$, contained in $\mathcal{U}$, such that

$$
e(x) \leqslant e\left(x_{v}(1)\right)-\phi\left(x_{v}(1)\right)+\phi(x),
$$

for every control $v(\cdot) \in \mathcal{V}$. Moreover it can be easily seen by definition of the distance function, that

$$
e\left(x_{v}(1)\right) \leqslant \int_{0}^{1} \sum_{i=1}^{m} v_{i}(t)^{2} d t .
$$

Therefore we obtain

$$
e(x) \leqslant \int_{0}^{1} \sum_{i=1}^{m} v_{i}(t)^{2} d t-\phi\left(x_{v}(1)\right)+\phi(x),
$$

for every control $v(\cdot) \in \mathcal{V}$. In particular, this means that $u(\cdot)$ is a solution of the minimization problem

$$
\min _{v \in \mathcal{V}}\left(\int_{0}^{1} \sum_{i=1}^{m} v_{i}(t)^{2} d t-\phi\left(x_{v}(1)\right)+\phi(x)\right) .
$$

Hence $u(\cdot)$ is a critical point of the function

$$
v(\cdot) \in \mathcal{V} \mapsto C(v(\cdot))-\phi\left(E_{x_{0}}(v(\cdot))\right)+\phi(x),
$$

and thus

$$
d C(u(\cdot))-\zeta . d E_{x_{0}}(u(\cdot))=0 .
$$

This leads to the existence of a normal extremal lift $\left(x_{u}(\cdot), p_{u}(\cdot),-\frac{1}{2}, u(\cdot)\right)$ such that $\left(x_{u}(1), p_{u}(1)\right)=\left(x, \frac{1}{2} \zeta\right)$. In particular, uniqueness of a minimizing path joining $x_{0}$ to $x$ follows.

Th. 1.1 is a straightforward consequence of Prop. 2.3 together with Th. 2.1. 


\section{Proof of Theorem 1.2}

\subsection{The limiting sub-differential}

Let $M$ be a smooth manifold of dimension $n$ and $\Omega$ be an open subset of $M$. Let $f: \Omega \rightarrow \mathbb{R}$ be a continuous function on $\Omega$; we call limiting sub-differential of the function $f$ at the point $x \in \Omega$ the subset of $T_{x}^{*} M$ defined by

$$
\partial_{L} f(x):=\left\{\lim \zeta_{n} \mid \zeta_{n} \in \partial_{P} f\left(x_{n}\right), x_{n} \rightarrow x\right\} .
$$

As the proximal sub-differential, the limiting sub-differential of $f$ at $x$ depends only on the local behavior of $f$ near $x$. Moreover by construction, $\partial_{L} f(x)$ is a closed subset of $T_{x}^{*} M$ which contains $\partial_{P} f(x)$, which is not necessarily convex and which may be empty. In some situations, the limiting sub-differential of $f$ at $x$ can be proven to be nonempty; the result is as follows.

Proposition 3.1. Let $x \in \Omega$. If there exists a Lipschitz continuous $\phi$ defined in a neighborhood of $x$ such that $f-\phi$ attains a local minimum at $x$, then $\partial_{L} f(x)$ is nonempty.

Proof. Without loss of generality, we can assume to be in $\mathbb{R}^{n}$. By assumption, the function $f-\phi$ attains a local minimum at $x$; this implies that $0 \in \partial_{L}(f-$ $\phi)(x)$. By the sum rule on limiting sub-differentials (see [6, Proposition $10.1 \mathrm{p}$. $62]$ ), the function $-\phi$ being Lipschitz continuous, there holds

$$
\partial_{L}(f-\phi)(x) \subset \partial_{L} f(x)+\partial_{L}(-\phi)(x),
$$

and hence $\partial_{L} f(x)$ is necessarily nonempty.

This proposition will be the key result to prove Th. 1.2. Notice that there exist some continuous functions $f: \mathbb{R}^{n} \rightarrow \mathbb{R}, n \geqslant 2$, such that their limiting sub-differential is empty on a subset of positive Lebesgue measure. However if $n=1$, it can be proven that the limiting sub-differential of any continuous function $f: \mathbb{R} \rightarrow \mathbb{R}$ is nonempty almost everywhere. Our proof of Th. 1.2 for corank-one distributions is in some way related to this latter result, but is not a consequence of it.

\subsection{Application to the proof of Theorem 1.2}

In what follows, we denote $e(\cdot):=d_{S R}\left(x_{0}, \cdot\right)^{2}$.

Proposition 3.2. Let $x \in M$ such that $\partial_{L} e(x) \neq \emptyset$ and let $\zeta \in \partial_{L} e(x)$. Then there exists a minimizing trajectory joining $x_{0}$ to $x$ which admits a normal extremal lift $\left(x(\cdot), p(\cdot),-\frac{1}{2}, u(\cdot)\right)$ such that $p(1)=\frac{1}{2} \zeta$.

Proof. By definition of the limiting sub-differential, there exists a sequence $\left(x_{n}\right)_{n \in \mathbb{N}}$ of points in $M$ converging to $x$ and a sequence $\left(\zeta_{n}\right)_{n \in \mathbb{N}} \in \partial_{P} e\left(x_{n}\right)$ such that $\lim \zeta_{n}=\zeta$. For each integer $n$, we denote by $u_{n}(\cdot)$ a minimizing control joining $x_{0}$ to $x_{n}$, and by $x_{u_{n}}(\cdot)$ its associated trajectory. From Prop. 
2.3 , for each integer $n$, we know that $x_{u_{n}}(\cdot)$ admits a normal extremal lift $\left(x_{u_{n}}(\cdot), p_{u_{n}}(\cdot),-\frac{1}{2}, u_{n}(\cdot)\right)$ such that $p_{u_{n}}(1)=\frac{1}{2} \zeta_{n}$. Since the sub-Riemannian distance is continuous, the sequence of controls $\left(u_{n}(\cdot)\right)_{n \in \mathbb{N}}$ is clearly bounded in $L^{2}\left([0,1], \mathbb{R}^{m}\right)$, and then up to a subsequence, it converges towards an element $u(\cdot)$ for the weak $L^{2}$-topology. As a consequence, since the end-point mapping $E_{x_{0}}$ is continuous for the weak $L^{2}$-topology (see [9] for a proof), we deduce, passing to the limit, that $E_{x_{0}}(u(\cdot))=x$. Furthermore, up to a subsequence the sequence $\left(x_{u_{n}}(\cdot)\right)_{n \in \mathbb{N}}$ converges uniformly towards a minimizimg path $x_{u}($.$) .$ This implies that the sequence $\left(p_{u_{n}}(\cdot)\right)_{n \in \mathbb{N}}$ converges uniformly towards some $p_{u}(\cdot)$, where $p_{u}(\cdot)$ is an adjoint vector associated to the trajectory $x_{u}(\cdot)$, and $p_{u}(1)=\frac{1}{2} \lim _{n \rightarrow \infty} \zeta_{n}$. Finally the quadruple $\left(x_{u}(\cdot), p_{u}(\cdot),-\frac{1}{2}, u(\cdot)\right)$ is a normal extremal lift of $x_{u}(\cdot)$.

Analogously to Th. 2.1, we have the following result.

Proposition 3.3. If the distribution is everywhere of corank one, then $\partial_{L} e(x) \neq$ $\emptyset$ for almost every $x \in M$.

Proof. In what follows, our point of view being local, we can assume to work in $\mathbb{R}^{n}$. Denote by $P$ the set of points $x$ of $M$ such that

$$
\liminf _{y \rightarrow x} \frac{e(y)-e(x)}{\|y-x\|}=-\infty .
$$

We have $M=P \cup P^{c}$, where $P^{c}$ denotes the complement of the set $P$ in $M$. Note that if $x \in P^{c}$ then there exists $\alpha \in \mathbb{R}$ such that $\liminf _{y \rightarrow x} \frac{e(y)-e(x)}{\|y-x\|}=\alpha$, which means that there exists a neighborhood $\mathcal{V}$ of $x$ such that

$$
e(y) \geqslant e(x)+(\alpha-1)\|y-x\|, \quad \forall y \in \mathcal{V} .
$$

We infer that the function $e$ has a Lipschitz continuous support function at $x$ and hence from Prop. 3.1 that $\partial_{L} e(x)$ is nonempty. The rest of the proof is devoted to show that the set $\partial_{L} e(x)$ is nonempty for almost every point $x \in P$. We argue by contradiction: denote by $A$ the subset of $P$ where the limiting sub-differential of $f$ is empty, and suppose that $\mu(A)>0$.

For all $x \in M$, let $\nu(x)$ denote a vector of $T_{x} M$ transverse to the distribution $\Delta(x)$. We may assume the vector field $\nu(\cdot)$ to be smooth on $M$. Let us consider integral curves of the differential system

$$
\dot{y}(t)=\nu(y(t)) .
$$

From Fubini's theorem, there exists an interval $I \subset \mathbb{R}$ and an integral curve $(y(t))_{t \in I}$ of $(6)$ such that the set

$$
T:=\{t \in I \mid y(t) \in A\},
$$

satisfies $\lambda(T)>0$, where $\lambda$ denotes the Lebesgue measure on $\mathbb{R}$. We are going to prove that some $\bar{t} \in I$ is the limit of local minima of the function $e(\cdot)$ restricted to the curve $y(t)$. To this aim we need different lemmas. 
Lemma 3.4. For all $x \in M$, there exist a neighborhood $\mathcal{V}_{x}$ of $x$ in $M$, a neighborhood $U_{x}$ of 0 in $T_{x}^{*} M$, and a submanifold $D_{x}$ of codimension 1 in $M$, such that

$$
\mathcal{V}_{x} \cap D_{x} \subset \exp _{x}\left(U_{x}\right) .
$$

Proof. Clearly the mapping $\exp _{x}$ is smooth on its domain of definition, and its differential at 0 , denoted $d \exp _{x}(0)$, can be computed as

$$
d \exp _{x}(0) . \delta p_{0}=\delta x(1),
$$

where $(\delta x(\cdot), \delta p(\cdot))$ is the solution of the linearized system of system (5) at the equilibrium point $(x, 0)$, such that $\delta x(0)=0$ and $\delta p(0)=\delta p_{0}$. This linearized system writes

$$
\delta \dot{x}(t)=\sum_{i=1}^{n-1}\left\langle\delta p(t), f_{i}(x)\right\rangle f_{i}(x), \quad \delta \dot{p}(t)=0,
$$

and thus $\delta p(t)$ is constant, equal to $\delta p_{0}$, whence

$$
\delta x(1)=\sum_{i=1}^{n-1}\left\langle\delta p_{0}, f_{i}(x)\right\rangle f_{i}(x) .
$$

Therefore the mapping $\exp _{x}$ has rank $n-1$ at the point 0 , and the conclusion follows.

For each $x \in M$, let $\left(p_{i}^{*}(x)\right)_{i=1, \ldots, n}$ denote the dual basis in $T_{x}^{*} M$ of the basis $\left(f_{1}(x), \ldots, f_{n-1}(x), \nu(x)\right)$ in $T_{x} M$. We define the mapping $\Phi: I \times O \rightarrow M$, where $O$ is a neighborhood of 0 in $\mathbb{R}^{n-1}$, by the formula

$$
\Phi\left(t, \alpha_{1}, \ldots, \alpha_{n-1}\right):=\exp _{y(t)}\left(\sum_{i=1}^{n-1} \alpha_{i} p_{i}^{*}(y(t))\right) .
$$

Using (7), it is quite easy to see that, for all $t_{0} \in I$, the mapping $\Phi$ is a local diffeomorphism at $\left(t_{0}, 0\right)$. Thus the following lemma is straightforward.

Lemma 3.5. Let $t_{0} \in T$. There exist a neighborhood $\mathcal{V}$ of $y\left(t_{0}\right)$ in $M$ and a

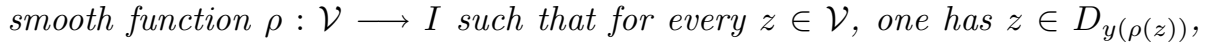
and such that for every $t \in T$ with $y(t) \in \mathcal{V}$, there holds $\rho(y(t))=t$. Moreover, there exists a real number $\delta>0$ such that

$$
|e(z)-e(y(\rho(z)))| \leqslant \delta\|z-y(\rho(z))\| .
$$

for all $z \in \mathcal{V}$.

Define the continuous function $g: I \rightarrow \mathbb{R}$ by $g(t):=e(y(t))$.

Lemma 3.6. There exists $\bar{t} \in T$ and a sequence $\left(t_{n}\right)_{n \in \mathbb{N}}$ of I converging towards $\bar{t}$, such that the function $g$ attains a local minimum at $t_{n}$, for every integer $n$. 
Proof. We argue by contradiction. If the conclusion of the lemma does not hold, this means that for every $t \in T$, there exists a neighborhood $V_{t}$ of $t$ in $I$ on which $g$ is monotonous. In particular $g$ has bounded variations on $V_{t}$, and hence $g$ is differentiable almost everywhere in $V_{t}$. On the other hand, since the set $T$ has positive Lebesgue measure, there exists $t \in T$ such that $\lambda(V \cap T)>0$ for any neighborhood $V$ of $t$ in $I$. Hence, this proves that for such a $t \in T$, the function $g$ is differentiable almost everywhere in $V_{t}$ which has positive measure. Fix some $s \in V_{t}$ where $g$ is differentiable. As a consequence, there exists some Lipschitz continuous function $\psi: I \rightarrow \mathbb{R}$ such that

$$
\psi(s)=g(s), \text { and } \psi\left(s^{\prime}\right) \leqslant g\left(s^{\prime}\right), \quad \forall s^{\prime} \in I .
$$

On the other hand, by Lemma 3.5, there exists a neighborhood $\mathcal{V}$ of $y(s)$ in $M$ and a smooth function $\rho: \mathcal{V} \rightarrow I$ which satisfy the property given in the lemma. By (8), we deduce that for any $x \in \mathcal{V}$, there holds

$$
\begin{aligned}
e(x) & \geqslant e(y(\rho(x)))-\delta\|x-y(\rho(x))\| \\
& \geqslant \phi_{s}(\rho(x))-\delta\|x-y(\rho(x))\| .
\end{aligned}
$$

Therefore if we define locally $\phi(x):=-\delta\|x-y(\rho(x))\|$, the function $e-\psi \circ \rho-\phi$ attains a local minimum at $y(s)$. Since $\psi \circ \rho$ and $\phi$ are Lipschitz continuous, the sum rule on limiting sub-differentials (see [6, Prop. 10.1 p. 62]) implies that

$$
0 \in \partial_{L}(e-\psi \circ \rho-\phi)(y(s)) \subset \partial_{L} e(y(s))+\partial_{L}(-\psi \circ \rho-\phi)(y(s)) .
$$

Hence there exists $\zeta \in \partial_{L} e(y(s))$ and $\zeta^{\prime} \in \partial_{L}(-\psi \circ \rho-\phi)(y(s))$ such that $0=\zeta+\zeta^{\prime}$ Which proves that $\partial_{L} e(y(s))$ is nonempty and which contradicts the fact that $y(s) \in A$.

Lemma 3.7. There exists some constant $K>0$ such that for every integer $n$, the limiting sub-differential $\partial_{L} e\left(y\left(t_{n}\right)\right)$ contains an element with norm less than $K$.

Proof. By construction of the sequence $\left(t_{n}\right)_{n \in \mathbb{N}}$, for every integer $n$ the function $g$ attains a minimum at $t_{n}$. This means that there exists an interval $\left(a_{n}, b_{n}\right)$ containing $t_{n}$ such that

$$
\forall t \in\left(a_{n}, b_{n}\right) \quad g(t) \geqslant g\left(t_{n}\right) .
$$

On the other hand, by Lemma 3.5, there exists a neighborhood $\mathcal{V}$ of $y(\bar{t})$ such that for $n$ large enough, any $x$ close enough to $y\left(t_{n}\right)$ belongs to $D_{y(\rho(x))}$ where $\rho(x) \in\left(a_{n}, b_{n}\right)$. By $(8)$, we deduce that for $x$ close enough to $y\left(t_{n}\right)$, there holds

$$
\begin{aligned}
e(x) & \geqslant e(y(\rho(x)))-\delta\|x-y(\rho(x))\| \\
& \geqslant e\left(y\left(t_{n}\right)\right)-\delta\|x-y(\rho(x))\| .
\end{aligned}
$$

Therefore as before, if we define locally $\phi(x):=-\delta\|x-y(\rho(x))\|$, the function $e-\phi$ attains a local minimum at $y\left(t_{n}\right)$. Since $\phi$ is Lipschitz continuous, the sum rule on limiting sub-differentials implies that

$$
0 \in \partial_{L}(e-\phi)\left(y\left(t_{n}\right)\right) \subset \partial_{L} e\left(y\left(t_{n}\right)\right)+\partial_{L}(-\phi)\left(y\left(t_{n}\right)\right) .
$$


Hence there exists $\zeta \in \partial_{L} e\left(y\left(t_{n}\right)\right)$ and $\zeta^{\prime} \in \partial_{L}(-\phi)\left(y\left(t_{n}\right)\right)$ such that $0=\zeta+\zeta^{\prime}$. Finally $\|\zeta\|=\left\|\zeta^{\prime}\right\|$ where $\left\|\zeta^{\prime}\right\|$ is less than the Lipschitz constant of the function $\phi$. This concludes the proof of the lemma.

Returning to the proof of Prop. 3.3, we infer easily that $\partial_{L} e(y(\bar{t}))$ is nonempty. This yields a contradiction with the fact that $y(\bar{t}) \in A$, and ends the proof of the proposition.

Propositions 3.2 and 3.3 imply the existence of a subset $N$ of full Lebesgue measure in $M$ such that, for every $x \in N$, there exists a minimizing trajectory steering $x_{0}$ to $x$ and having a normal extremal lift. Let $N_{2}:=N \backslash \mathcal{C}\left(x_{0}\right)$. It is the set of points $x \in M$ which are not conjugate to $x_{0}$, and such that there exists a minimizing path $x(\cdot)$ joining $x_{0}$ to $x$ and having a normal extremal lift. Remark 1.7 implies that the trajectory $x(\cdot)$ is moreover nonsingular. From Remark 1.6 it is clear that $N_{2}$ is of full Lebesgue measure in $M$. This ends the proof of Th. 1.2.

\section{Consequences and open questions}

In what follows, we assume that Chow's condition holds, and that the manifold $M$ is complete for the sub-Riemannian distance. Let $x_{0} \in M$ be fixed.

\subsection{A formula for the sub-Riemannian distance}

From Th. 1.1, there exists a dense subset $N_{1}$ of $M$ such that every point of $N_{1}$ can be joined from $x_{0}$ by a unique minimizing trajectory, which moreover admits a normal extremal lift. This yields the following result.

Corollary 4.1. For all point $x \in N_{1}$ one has

$$
d_{S R}\left(x_{0}, x\right)=\inf \left\{\left(2 H_{1}\left(x_{0}, p\right)\right)^{1 / 2} \mid p \in U \text { s.t. } \exp _{x_{0}}(p)=x\right\} .
$$

Remark 4.1. Actually Th. 1.1 implies that for every $x \in N_{1}$ there exists a unique $p \in U$ such that the above infimum is attained.

As a consequence, we deduce that the function $g: M \rightarrow \mathbb{R} \cup\{\infty\}$ defined by

$$
g(x):=\inf \left\{\left(2 H_{1}\left(x_{0}, p\right)\right)^{1 / 2} \mid p \in U \text { s.t. } \exp _{x_{0}}(p)=x\right\},
$$

for all $x \in M$, coincides with the mapping $d_{S R}\left(x_{0}, \cdot\right)$ on a dense subset of the manifold $M$. In particular, since $g$ is continuous on $M$, there holds

$$
d_{S R}\left(x_{0}, x\right)=\inf \left\{\lim g\left(x_{n}\right) \mid x_{n} \rightarrow x\right\}
$$

for all $x \in M$. 
Remark 4.2. If the sub-Riemannian distance to $x_{0}$ is Lipschitz continuous outside $x_{0}$, then from Prop 2.2 the limiting sub-differentials of $d_{S R}\left(x_{0}, \cdot\right)$ are always nonempty; hence the set of points $x$ of $M$ such that every minimizing trajectory joining $x_{0}$ to $x$ is strictly singular, is empty. The converse is false; a counterexample is given by the so-called Martinet flat case, see [2]. To get a converse statement, the assumption has to be strengthened as follows: if there does not exist any nontrivial singular minimizing trajectory, then $d_{S R}\left(x_{0}, \cdot\right)$ is Lipschitz continuous outside $x_{0}$, see [1].

\subsection{On the sub-Riemannian wave-front and sphere}

The following result is a direct consequence of Th. 1.1.

Corollary 4.2. The sub-Riemannian wave-front $W_{S R}\left(x_{0}, r\right)$ is connected, for all $r>0$.

Proof. Using notations of Remark 1.5, and from Th. 1.1, we have the inclusions

$$
\exp _{x_{0}}\left(U_{r}\right) \subset W_{S R}\left(x_{0}, r\right) \subset \overline{\exp _{x_{0}}\left(U_{r}\right)},
$$

where $U_{r}$ is diffeomorphic to $\mathcal{S}^{m-1} \times \mathbb{R}^{n-m}$, and thus is connected. The conclusion follows readily.

Proposition 4.3. If the distribution $\Delta$ is everywhere of corank one, then the sub-Riemannian wave-front $W_{S R}\left(x_{0}, r\right)$, and thus the sub-Riemannian sphere $S_{S R}\left(x_{0}, r\right)$, has Lebesgue measure zero, for all $r>0$.

Proof. It suffices to notice that the image by a locally lipschitzian mapping from $\mathbb{R}^{n}$ to $\mathbb{R}^{n}$ of a set of zero measure has zero measure, and to apply Th. 1.2.

\subsection{Sard type conjectures}

Let $\mathcal{A}$ (resp. $\mathcal{A}_{s}$ ) denote the set of points $x$ of $M$ such that every minimizing trajectory joining $x_{0}$ to $x$ is singular (resp. strictly singular). Obviously $\mathcal{A}_{s} \subset \mathcal{A}$. Th. 1.1 and 1.2 yield the following result.

Corollary 4.4. The subset $\mathcal{A}_{s}$ has an empty interior in $M$. In the case of a corank-one distribution the subset $\mathcal{A}$ has Lebesgue measure zero in $M$.

Let now $\mathcal{S}$ (resp. $\mathcal{S}_{\text {min }}$, resp. $\mathcal{S}_{\text {min }}^{\text {strict }}$ ) denote the set of points $x$ of $M$ such that there exists a singular trajectory (resp. a singular minimizing trajectory, resp. a strictly singular minimizing trajectory) steering $x_{0}$ to $x$. Notice that $\mathcal{S}$ is the set of critical values of the end-point-mapping $E_{x_{0}}$.

Corollary 4.5. The set $\mathcal{S}_{\text {min }}^{\text {strict }}$ has an empty interior in $M$.

Let $N_{3}$ be the set of points $x \in M$ such that there exists a unique minimizing path $x(\cdot)$ joining $x_{0}$ to $x$, which moreover admits a normal extremal lift, and such that $x$ is not conjugate to $x_{0}$. Notice that from Remark 1.7, the path $x(\cdot)$ is nonsingular. The set $N_{3}$ can be proven to be open in $M$; we formulate the following conjecture. 
Conjecture 4.6. The subset $N_{3}$ is of full Lebesgue measure in $M$. In particular, the set $\mathcal{S}_{\text {min }}$ has Lebesgue measure zero in $M$.

We end the paper with the following open question.

Conjecture 4.7. The end-point mapping satisfies Sard's property, i.e. the set $\mathcal{S}$ has Lebesgue measure zero in $M$.

This conjecture has been formulated and discussed, among others, in [7]. Up to now, it is still open, even in the case of a corank-one distribution.

Acknowledgement. The authors are very indebted to F. H. Clarke for useful discussions.

\section{References}

[1] A. Agrachev. Compactness for sub-Riemannian length-minimizers and subanalyticity. Rend. Sem. Mat. Univ. Politec. Torino, 56(4):1-12, 1998.

[2] A. Agrachev, B. Bonnard, M. Chyba, and I. Kupka. Sub-Riemannian sphere in Martinet flat case. ESAIM Control Optim. Calc. Var., 2:377448 (electronic), 1997.

[3] A. Bellaïche. Tangent space in sub-Riemannian geometry. Sub-Riemannian geometry, Birkhäuser, 1996.

[4] J.-M. Bismut. Large deviations and the Malliavin calculus. Progress in Mathematics 45, Birkhäuser, 1984.

[5] J. M. Borwein and D. Preiss. A smooth variational principle with applications to subdifferentiability and to differentiability of convex functions. Trans. Amer. Math. Soc., 303(2):517-527, 1987.

[6] F. H. Clarke, Yu. S. Ledyaev, R. J. Stern, and P. R. Wolenski. Nonsmooth Analysis and Control Theory. Graduate Texts in Mathematics, vol. 178. Springer-Verlag, New York, 1998.

[7] R. Montgomery. A tour of subriemannian geometries, their geodesics and applications. Mathematical Surveys and Monographs, 91. American Mathematical Society, Providence, RI, 2002.

[8] L. Pontryagin et al.. Théorie mathématique des processus optimaux. Eds Mir, Moscou, 1974.

[9] E. Trélat. Some properties of the value function and its level sets for affine control systems with quadratic cost. J. of Dyn. and Cont. Syst., 6(4):511$541,2000$. 